\title{
On Gromov-Hausdorff stability in a boundary rigidity problem
}

\author{
SERGEI IVANOV
}

Let $M$ be a compact Riemannian manifold with boundary. We show that $M$ is Gromov-Hausdorff close to a convex Euclidean region $D$ of the same dimension if the boundary distance function of $M$ is $C^{1}$-close to that of $D$. More generally, we prove the same result under the assumptions that the boundary distance function of $M$ is $C^{0}$-close to that of $D$, the volumes of $M$ and $D$ are almost equal, and volumes of metric balls in $M$ have a certain lower bound in terms of radius.

$53 \mathrm{C} 23$

\section{Introduction}

Let $M$ be a compact Riemannian manifold with boundary. For $x, y \in M$, we denote by $d_{M}(x, y)$ the Riemannian distance between $x$ and $y$, that is the length of a shortest curve connecting $x$ and $y$. The boundary distance function, denoted by $b d_{M}$, is the restriction of $d_{M}$ to $\partial M \times \partial M$.

In some cases $M$ is uniquely determined by $b d_{M}$ (up to an isometry fixing the boundary); such Riemannian manifolds $M$ are called boundary rigid. Michel [14] conjectured that every simple Riemannian manifold (that is, such that the boundary is strictly convex and all geodesics are minimizing and free of conjugate points) is boundary rigid. This conjecture is proved in dimension 2 by Pestov and Uhlmann [15] and in some partial cases in higher dimensions (see Michel [14], Gromov [12], Besson, Courtois and Gallot [4], Croke and Kleiner [9] and Burago and Ivanov [7]). In particular, it is shown in [7] that, if $M$ is a region in $\mathbb{R}^{n}$ with a Riemannian metric which is sufficiently close (in $C^{2}$ ) to the Euclidean metric $g_{e}$, then $M$ is boundary rigid. In other words, if a Riemannian metric $g$ on $D$ defines the same boundary distance function as some almost Euclidean metric $g^{\prime}$, then $g$ is isometric to $g^{\prime}$.

This raises the following stability question: if the boundary distance function of a metric $g$ is close to that of $g_{e}$ in a suitable topology, is $g$ necessarily close to $g_{e}$ in $C^{r}, r \geq 2$ (up to an isometry fixing the boundary)? The answer is known to be affirmative in a local variant of the question, namely under the assumption that the 
$C^{m}$-norm of $g$, for a suitable $m>r$, is a priori bounded (see Wang [18] and, for a more general result, Stefanov and Uhlmann [17]). However the global stability question (without further assumptions on $g$ ) remains open.

In this paper we give an affirmative answer to a weaker variant of this question, namely we show that $g$ is close to $g_{e}$ in the Gromov-Hausdorff topology. The assumptions on the boundary distance function are also relatively weak: it should be only $C^{1}$-close to the boundary distance function of the Euclidean metric. The precise statement is the following:

Theorem 1 Let $D \subset \mathbb{R}^{n}$ be a strictly convex compact region with a smooth boundary. Then for every $\varepsilon>0$ there exists $\delta>0$ such that the following holds. Let $M$ be a Riemannian manifold such that $\partial M=\partial D, b d_{M}$ is $C^{1}$-smooth on $\partial D \times \partial D \backslash \Delta$ where $\Delta$ is the diagonal of $\partial D \times \partial D$, and

$$
\left\|b d_{M}-b d_{D}\right\|_{C^{1}(\partial D \times \partial D \backslash \Delta)}<\delta .
$$

Then $d_{\mathrm{GH}}(M, D)<\varepsilon$ where $d_{\mathrm{GH}}$ is the Gromov-Hausdorff distance.

Here "strictly convex" means that $\partial D$ contains no straight line segment. We refer to Gromov [13, Section 3A] or Burago, Burago and Ivanov [5, Section 7.3] for the definition of the Gromov-Hausdorff distance. For the purposes of this paper, the following criterion is sufficient [5, Corollary 7.3.28]: for metric spaces $X$ and $Y$, one has $d_{\mathrm{GH}}(X, Y) \leq 2 \varepsilon$ if there is a map $f: X \rightarrow Y$ such that $f(X)$ is an $\varepsilon$-net in $Y$ (that is, the $\varepsilon$-neighborhood of $f(X)$ covers $Y$ ) and

$$
\left|d_{Y}\left(f(x), f\left(x^{\prime}\right)\right)-d_{X}\left(x, x^{\prime}\right)\right| \leq \varepsilon
$$

for all $x, x^{\prime} \in X$. Such maps are referred to as $\varepsilon$-approximations.

The boundary distance function $b d_{M}$ is not differentiable at the diagonal; this is why the theorem involves the $C^{1}$ norm on $\partial D \times \partial D \backslash \Delta$. Alternatively, one may require that $\left\|b d_{M}^{2}-b d_{D}^{2}\right\|_{C^{1}}$ is small and the metric tensors of $M$ and $D$ restricted to $\partial D$ are $C^{0}$-close to each other.

Theorem 1 is proved in Section 5. Here is a sketch of the proof. For simplicity, assume that $\partial M$ is strictly convex (a nonconvex boundary requires more technical details; see Section 5). Then the fact that $b d_{M}$ is $C^{1}$ implies that all geodesics in $M$ are minimizing. For such metrics, Santaló's integral geometric formula (cf Section 2.2) allows one to express the total volume of $M$ in terms of the boundary distance function and its derivatives. Applying this formula to $M$ and $D$ yields that $\operatorname{vol}(M) \approx \operatorname{vol}(D)$. Since all geodesics in $M$ are minimizing, the exponential map at every point is injective. Then Croke's local isoembolic inequality (cf Section 2.3) yields a uniform lower bound 
for volumes of metric balls in $M$ in terms of radii. With these observations, Theorem 1 follows from Theorem 2 (see below) which requires only $C^{0}$ closeness of the boundary distance functions but includes volume related assumptions.

For a set $A \subset M$ and $r>0$, we denote by $U_{r}(A)$ the metric $r$-neighborhood of $A$, that is,

$$
U_{r}(A)=\left\{x \in M: \operatorname{dist}_{M}(x, A)<r\right\}
$$

where $\operatorname{dist}_{M}(x, A)=\inf _{y \in A} d_{M}(x, y)$. By $B_{r}(x)$ we denote the metric ball of radius $r$ centered at $x \in M$, that is $B_{r}(x)=U_{r}(\{x\})$.

Theorem 2 Let $D \subset \mathbb{R}^{n}$ be a convex compact region and $\lambda>0$ a positive constant. Then for every $\varepsilon>0$ there exists $\delta=\delta(D, \lambda, \varepsilon)>0$ such that the following holds: if a compact Riemannian $n$-manifold $M$ with $\partial M=\partial D$ satisfies

(1) $\left|d_{M}(x, y)-d_{D}(x, y)\right|<\delta$ for all $x, y \in \partial M=\partial D$,

(2) $\operatorname{vol}\left(M \backslash U_{\delta}(\partial M)\right)<\operatorname{vol}(D)+\delta$,

(3) $\operatorname{vol}\left(B_{r}(x)\right) \geq \lambda r^{n}$ for all $x \in M$ and all $r \geq \delta$ such that $B_{r}(x) \cap \partial M=\varnothing$,

then $d_{\mathrm{GH}}(M, D)<\varepsilon$.

Remark 1.1 If $d_{M}(x, y) \geq d_{D}(x, y)$ for all $x, y \in \partial M=\partial D$, then $\operatorname{vol}(M) \geq \operatorname{vol}(D)$ and the equality $\operatorname{vol}(M)=\operatorname{vol}(D)$ implies that $M$ is isometric to $D$. This can be shown as follows. One may assume that $D$ is contained in the unit cube $I^{n}$. Replacing $D \subset I^{n}$ by $M$ yields a piecewise Riemannian manifold whose boundary is identified with $\partial I^{n}$ in such a way that the distances between opposite faces are no less than 1 . Then the Besicovitch inequality [3] (see also Gromov [12, Section 7.1]) implies that the volume of this space is at least 1 , and in the case of equality the space must be isometric to $I^{n}$. Thus Theorem 2 is in a sense a stability estimate in the equality case of the Besicovitch inequality.

The following example shows both volume assumptions in Theorem 2 are necessary.

Example 1.2 Let $D \subset \mathbb{R}^{n}$ be the standard unit ball and $D_{r}$ be a ball of radius $r \ll \delta$ with the same center. Remove $D_{r}$ from $D$ and replace it with either a big round $n$-dimensional sphere with a similar ball removed, or a closed-up cylinder $\left(\partial D_{r} \times[0, L]\right) \cup\left(D_{r} \times\{L\}\right)$ where $L \gg 1$. Smoothening the resulting piecewise Riemannian metric yields an example of $M$ such that $\left\|b d_{M}-b d_{D}\right\|_{C^{0}}<\delta$ but $d_{\mathrm{GH}}(M, D) \geq 1$. Only the second assumption of Theorem 2 is violated in the big sphere example, and only the third one in the cylinder example. 
Theorem 2 is proved in Section 3 and Section 4. In Section 3 we use special distancelike functions on $M$ to construct a Lipschitz map $\varphi: M \rightarrow \mathbb{R}^{n}$ which is volume nonincreasing and whose image approximates $D$. Then in Section 4 we show that $\varphi$ almost preserves distances up to a small additive term, and hence is an $\varepsilon$-approximation. The key points of the proof are assertion (3-7) of Proposition 3.1 and Lemma 4.4.

Remark 1.3 The first assumption in Theorem 2 can be replaced by a weaker one that does not require identifying $\partial M$ with $\partial D$ : there is a continuous map $F: \partial M \rightarrow \partial D$ of nonzero degree mod 2 such that $\left|d_{M}(x, y)-d_{\mathbb{R}^{n}}(F(x), F(y))\right|<\delta$ for all $x, y \in \partial M$. This is what is actually used in the proof, see Section 3 .

Acknowledgements Many ideas used in this paper arose from joint work with Dima Burago [7;6]. I am grateful to Anton Petrunin who suggested a simple proof of Lemma 3.3 and to Sergei Buyalo for his helpful remarks about the text.

The author was supported by the Dynasty foundation and RFBR grant number 09-0112130-ofi-m.

\section{Preliminaries}

In this section we state some results used throughout the paper.

\subsection{Area inequality}

Let $M^{n}$ and $M_{1}^{n_{1}}$ be Riemannian manifolds and $f: M \rightarrow M_{1}$ a Lipschitz map. By Rademacher's Theorem (see Federer [10, 3.1.6]), $f$ is differentiable almost everywhere on $M$. Let $x \in M$ be a point where $f$ is differentiable. The ( $n$-dimensional) Jacobian of $f$ at $x$, denoted by $J f(x)$, is the $n$-dimensional volume of the image of a unit cube in $T_{x} M$ under the derivative $d_{x} f: T_{x} M \rightarrow T_{f(x)} M_{1}$. We need the following inequality which is an easy corollary of the area formula for Lipschitz maps [10, 3.2.3].

Proposition 2.1 For every measurable set $A \subset M$, one has

$$
\operatorname{vol}_{n}(f(A)) \leq \int_{A} J f(x) d \operatorname{vol}_{n}(x)
$$

where $\operatorname{vol}_{n}$ denotes the $n$-dimensional Hausdorff measure. In particular, if $J f \leq 1$ a.e., then $f$ does not increase $n$-dimensional volumes. 


\subsection{Santaló's formula}

In order to deduce Theorem 1 from Theorem 2, we need some integral geometry in the space of geodesics. Let $M$ be a compact Riemannian manifold with boundary. We denote by $S M$ the unit tangent bundle of $M$. For $p \in \partial M$, denote by $v(p)$ the unit inner normal to $\partial M$ and by $S_{p}^{+} M$ a hemisphere in $T_{p} M$ defined by

$$
S_{p}^{+} M=\left\{v \in S_{p} M:\langle v, v(p)\rangle \geq 0\right\} .
$$

By a geodesic in $M$ we mean a unit-speed curve in $M$ which is a geodesic of the Riemannian metric and does not have points on $\partial M$ except possibly endpoints. For a unit tangent vector $v \in S_{p} M$, we denote by $\gamma_{v}$ the maximal geodesic $\gamma:[0, a] \rightarrow M$ or $\gamma:[0,+\infty) \rightarrow M$ defined by initial data $\gamma(0)=p$ and $\dot{\gamma}(0)=v$. The length of $\gamma_{v}$ is denoted by $\ell(v)$ or $\ell_{M}(v)$.

The standard Liouville measure $\mu_{L}$ on $S M$ is defined by

$$
\mu_{L}(A)=\int_{M} \operatorname{vol}_{S_{p} M}\left(A \cap S_{p} M\right) d \operatorname{vol}_{M}(p)
$$

for every measurable set $A \subset S M$, where $\operatorname{vol}_{S_{p}} M$ is the standard (n-1)-dimensional volume on the (Euclidean) sphere $S_{p} M$. In particular, $\mu_{L}(S M)=\omega_{n-1} \operatorname{vol}(M)$ where $\omega_{n-1}$ is the volume of the unit sphere in $\mathbb{R}^{n}$. The Liouville measure of a set invariant under a geodesic flow can be recovered from its slice by the boundary (see Santaló [16, Sections 19.4-19.5], Michel [14] or Gromov [12, page 60]), namely the following holds:

Proposition 2.2 Let $A \subset \bigcup_{p \in \partial M} S_{p}^{+} M$ be a Borel measurable set and let $\Phi(A) \subset$ $S M$ be the trajectory of $A$ under the geodesic flow (that is, $\Phi(A)$ is the set of velocity vectors of all geodesics of the form $\gamma_{v}$ where $v \in A$ ). Then

$$
\mu_{L}(\Phi(A))=\int_{\partial M} d \operatorname{vol}_{\partial M}(p) \int_{A \cap S_{p}^{+} M} \ell(v) \cos \angle(v, v(p)) d \operatorname{vol}_{S_{p} M}(v) .
$$

Remark 2.3 If a geodesic $\gamma_{v}$ (where $v \in S_{p}^{+} M$ ) is a unique minimizing geodesic between boundary points $p$ and $q$, then the angle $\angle(v, v(p))$ is uniquely determined by the derivative at $p$ of the function $b d_{M}(\cdot, q)$, cf Lemma 5.1. Thus Proposition 2.2 implies that the total volume of a simple Riemannian manifold $M$ is uniquely determined by $b d_{M}$. 


\subsection{Local isoembolic inequality}

M Berger [2] proved that the volume of a closed Riemannian manifold $M^{n}$ is bounded below by the $n$-th power of the injectivity radius times a constant depending on $n$ (the equality is attained when $M$ is a round $n$-sphere). This fact is often referred to as the isoembolic inequality. We need the following "local" version of this inequality, proved by C Croke.

Proposition 2.4 [8, Proposition 14] Let $M^{n}$ be a complete Riemannian manifold, possibly with boundary. Let $x \in M$ and $r>0$ be such that $B_{r}(x) \cap \partial M=\varnothing$ and every geodesic segment contained in $B_{r}(x)$ is minimizing (ie is a shortest path between its endpoints). Then

$$
\operatorname{vol}\left(B_{r}(x)\right) \geq c r^{n}
$$

for some explicit constant $c=c(n)>0$.

Remark 2.5 In [8], the result is stated only for boundaryless manifolds, but the proof uses only the fact that the ball in question does not reach the boundary. Indeed, (2-1) follows immediately from the identity $\operatorname{vol}\left(B_{r}(x)\right)=\int_{0}^{r} \operatorname{vol}_{n-1}\left(\partial B_{t}(x)\right) d t$ (which holds for any complete Riemannian manifold $M, x \in M$ and $r>0$ such that $\left.B_{r}(x) \cap \partial M=\varnothing\right)$ and an isoperimetric inequality

$$
\frac{\operatorname{vol}_{n-1}\left(\partial B_{t}(x)\right)}{\operatorname{vol}\left(B_{t}(x)\right)^{(n-1) / n}} \geq \operatorname{const}(n)
$$

[8, Theorem 11] which holds for any region (in place of $\left.B_{t}(x)\right)$ where all geodesics are minimizing.

\section{Distance-like coordinates}

This section is the first part of the proof of Theorem 2. Here we construct a Lipschitz map $\varphi: M \rightarrow \mathbb{R}^{n}$ (our would-be Gromov-Hausdorff approximation) and establish some of its technical properties (summarized in Proposition 3.1).

Let $M$ satisfy the assumptions of Theorem 2 for a small $\delta$. We fix $D$ and $\lambda$ and omit dependence on them in our notation. We denote by $\varepsilon(\delta)$ various quantities depending on $\delta$ and tending to 0 as $\delta \rightarrow 0$. In this notation, the assertion of Theorem 2 is that $d_{\mathrm{GH}}(M, D)<\varepsilon(\delta)$. The notation $A \approx B$ is an abbreviation for $|A-B|<\varepsilon(\delta)$. Denote $M^{\prime}=M \backslash U_{\delta}(\partial M)$. 
To avoid confusion in notation caused by identifying $\partial M$ with $\partial D$, we replace the first assumption of Theorem 2 by the following: there is a continuous map $F: \partial M \rightarrow \partial D$ of nonzero degree mod 2 such that

$$
\left|d_{M}(x, y)-d_{\mathbb{R}^{n}}(F(x), F(y))\right|<\delta \text { for all } x, y \in \partial M .
$$

We fix such a map $F$ for the rest of this section.

For a unit vector $v \in S^{n-1} \subset \mathbb{R}^{n}$, define a linear function $L_{v}: \mathbb{R}^{n} \rightarrow \mathbb{R}$ by

$$
L_{v}(x)=\langle x, v\rangle
$$

where $\langle\cdot, \cdot\rangle$ is the scalar product in $\mathbb{R}^{n}$, and a function $\varphi_{v}: M \rightarrow \mathbb{R}$ by

$$
\varphi_{v}(x)=\inf _{y \in \partial M}\left\{d_{M}(x, y)+L_{v}(F(y))\right\}
$$

where $F$ is the map from (3-1). Note that this function is 1 -Lipschitz on $M$ since it is a pointwise infimum of 1 -Lipschitz functions. Define a map $\varphi: M \rightarrow \mathbb{R}^{n}$ by

$$
\varphi(x)=\left(\varphi_{e_{1}}(x), \ldots, \varphi_{e_{n}}(x)\right)
$$

where $\left(e_{1}, \ldots, e_{n}\right)$ is the standard basis of $\mathbb{R}^{n}$. Obviously $\varphi$ is $n$-Lipschitz. Since the coordinate functions of $\varphi$ are 1-Lipschitz, its Jacobian at any point of differentiability is no greater than 1 . Therefore $\varphi$ is volume nonincreasing.

Our ultimate goal is to show that $\varphi$ is an $\varepsilon(\delta)$-approximation of a small neighborhood of $D$ in $\mathbb{R}^{n}$. In this section we prove the following proposition.

Proposition 3.1 For every unit vector $v \in \mathbb{R}^{n}$ the following holds.

(3-3) For every $x \in M$ there is a point $y \in \partial M$ such that $\varphi_{v}(x)=\varphi_{v}(y)+d_{M}(x, y)$.

(3-4) $|\varphi(x)-\varphi(y)| \approx d_{M}(x, y)$ for all $x, y \in \partial M$.

(3-5) $\operatorname{vol}(\varphi(E)) \geq \operatorname{vol}(E)-\varepsilon(\delta)$ for every measurable $E \subset M^{\prime}$.

(3-6) $\varphi(M)$ and $\varphi(\partial M)$ are within Hausdorff distance $\varepsilon(\delta)$ from $D$ and $\partial D$, resp.

(3-7) $\varphi_{v}(x) \approx L_{v}(\varphi(x))$ for all $x \in M$.

The proof of Proposition 3.1 occupies the rest of this section. Most of the assertions are nearly trivial; only (3-7) requires some work.

Proof of (3-3) Fix $x \in M$ and let $y \in \partial M$ be a point where the infimum in (3-2) is attained. Then $\varphi_{v}(x)=d_{M}(x, y)+L_{v}(F(y))$. Since $\varphi_{v}$ is 1-Lipschitz, it follows that $\varphi_{v}(y) \geq L_{v}(F(y))$. On the other hand,

$$
\varphi_{v}(y) \leq d_{M}(y, y)+L_{v}(F(y))=L_{v}(F(y))
$$

by the definition of $\varphi_{v}$. Thus $\varphi_{v}(y)=L_{v}(F(y))$ and (3-3) follows. 
Proof of (3-4) For every $x \in \partial M$ and every unit vector $v \in \mathbb{R}^{n}$ we have

$$
\left|\varphi_{v}(x)-L_{v}(F(x))\right| \leq \delta
$$

where $F$ is the map from (3-1). Indeed, for every $y \in \partial M$,

$$
d_{M}(x, y)+L_{v}(F(y)) \geq d_{\mathbb{R}^{n}}(F(x), F(y))+L_{v}(F(y))-\delta \geq L_{v}(F(x))-\delta
$$

since $L_{v}$ is 1 -Lipschitz. Hence $\varphi_{v}(x) \geq L_{v}(F(x))-\delta$. On the other hand, substituting $y=x$ under the infimum in (3-2) yields that $\varphi_{v}(x) \leq L_{v}(F(x))$, and (3-8) follows. Since $\left(L_{e_{1}}, \ldots, L_{e_{n}}\right)=\mathrm{id}_{\mathbb{R}^{n}},(3-8)$ implies

$$
|\varphi(x)-F(x)| \leq n \delta \quad \text { for all } x \in \partial M .
$$

This and (3-1) imply that

$$
|\varphi(x)-\varphi(y)| \approx|F(x)-F(y)| \approx d_{M}(x, y)
$$

for all $x, y \in \partial M$.

Proof of (3-5) By (3-9), $\varphi(\partial M) \subset U_{n \delta}(F(\partial M))=U_{n \delta}(\partial D)$ and moreover $\left.\varphi\right|_{\partial M}$ is homotopic to $F$ in $U_{n \delta}(\partial D)$. Therefore $\varphi$ has degree 1 over any point of $D \backslash U_{n \delta}(\partial D)$, hence $\varphi(M) \supset D \backslash U_{n \delta}(\partial D)$. Furthermore,

$$
\varphi\left(M \backslash M^{\prime}\right)=\varphi\left(U_{\delta}(\partial M)\right) \subset U_{2 n \delta}(\partial D),
$$

since $\varphi$ is $n$-Lipschitz. Hence

$$
\varphi\left(M^{\prime}\right) \supset D \backslash U_{2 n \delta}(\partial D) .
$$

Since $\varphi$ is volume nonincreasing, we have

$$
\operatorname{vol}\left(M^{\prime}\right) \geq \operatorname{vol}\left(\varphi\left(M^{\prime}\right)\right) \geq \operatorname{vol}\left(D \backslash U_{2 n \delta}(\partial D)\right)>\operatorname{vol}(D)-\varepsilon(\delta)>\operatorname{vol}\left(M^{\prime}\right)-\varepsilon(\delta)
$$

by (3-11) and the second assumption of Theorem 2. Let $E \subset M^{\prime}$ be a measurable set. Then

$$
\operatorname{vol}(\varphi(E))+\operatorname{vol}\left(\varphi\left(M^{\prime} \backslash E\right)\right) \geq \operatorname{vol}\left(\varphi\left(M^{\prime}\right)\right)>\operatorname{vol}\left(M^{\prime}\right)-\varepsilon(\delta) .
$$

On the other hand, $\operatorname{vol}\left(\varphi\left(M^{\prime} \backslash E\right)\right) \leq \operatorname{vol}\left(M^{\prime} \backslash E\right)$ since $\varphi$ is volume nonincreasing. Hence $\operatorname{vol}(\varphi(E))>\operatorname{vol}\left(M^{\prime}\right)-\operatorname{vol}\left(M^{\prime} \backslash E\right)-\varepsilon(\delta)=\operatorname{vol}(E)-\varepsilon(\delta)$.

Proof of (3-6) The assertion about $\varphi(\partial M)$ follows from (3-9) and the fact that $F(\partial M)=\partial D$. By (3-11), $D$ is contained in a small neighborhood of $\varphi(M)$. It remains to show that $\varphi(M)$ is contained in a small neighborhood of $D$. 
Let $p \in M$ and $r=\operatorname{dist}_{\mathbb{R}^{n}}(\varphi(p), D)$. We are to prove that $r<\varepsilon(\delta)$. Suppose that $r>4 n \delta$ and consider a metric ball $B=B_{r / 2 n}(p)$. Since $\varphi$ is $n$-Lipschitz, we have

$$
\varphi(B) \subset B_{r / 2}(\varphi(p)) \subset \mathbb{R}^{n} \backslash U_{2 n \delta}(D),
$$

hence $B \subset M^{\prime}$ by (3-10). Hence by (3-5) and the third assumption of Theorem 2 we have

$$
\operatorname{vol}(\varphi(B))>\operatorname{vol}(B)-\varepsilon(\delta) \geq \lambda(r / 2 n)^{n}-\varepsilon(\delta) .
$$

This and (3-11) imply that

$\operatorname{vol}\left(M^{\prime}\right) \geq \operatorname{vol}\left(\varphi\left(M^{\prime}\right)\right) \geq \operatorname{vol}\left(D \backslash U_{2 n \delta}(\partial U)\right)+\operatorname{vol}(\varphi(B))>\operatorname{vol}(D)+\lambda(r / 2 n)^{n}-\varepsilon(\delta)$.

On the other hand, $\operatorname{vol}\left(M^{\prime}\right)<\operatorname{vol}(D)+\varepsilon(\delta)$ by the second requirement of Theorem 2 . Therefore $\lambda(r / 2 n)^{n}<\varepsilon(\delta)$, hence $r<\varepsilon(\delta)$.

Proof of (3-7) We need one more construction and some lemmas.

Fix a unit vector $v \in \mathbb{R}^{n}$ and an orthonormal basis $\left(v_{1}, v_{2}, \ldots, v_{n}\right)$ in $\mathbb{R}^{n}$ such that $v_{1}=v$. Define a linear map $I: \mathbb{R}^{n} \rightarrow \mathbb{R}^{2 n}$ by

$$
I=\frac{1}{\sqrt{2}}\left(L_{e_{1}}, \ldots, L_{e_{n}}, L_{v_{1}}, \ldots, L_{v_{n}}\right)
$$

and a Lipschitz map $\Phi: M \rightarrow \mathbb{R}^{2 n}$ by

$$
\Phi=\frac{1}{\sqrt{2}}\left(\varphi_{e_{1}}, \ldots, \varphi_{e_{n}}, \varphi_{v_{1}}, \ldots, \varphi_{v_{n}}\right) .
$$

Observe that $I$ is a linear isometric embedding, $\Phi$ is a $2 n$-Lipschitz map and

$$
|\Phi(x)-I(F(x))| \leq 2 n \delta
$$

for all $x \in \partial M$ (by (3-8) and (3-9)). Therefore

$$
\Phi\left(M \backslash M^{\prime}\right)=\Phi\left(U_{\delta}(\partial M)\right) \subset U_{4 n \delta}(I(\partial M)) .
$$

Lemma 3.2 $\Phi$ does not increase $n$-dimensional volumes.

Proof The statement follows from the fact that the $2 n$ coordinate functions of $\Phi$ are $(1 / \sqrt{2})$-Lipschitz.

Indeed, let $p$ be a point of differentiability of $\Phi$ and let $Q$ be the pullback of the Euclidean structure of $\mathbb{R}^{2 n}$ by $d_{p} \Phi$. That is, $Q$ is a quadratic form on $T_{p} M$ defined by $Q(w)=\left|d_{p} \Phi(w)\right|^{2}$ for all $w \in T_{p} M$. Then

$$
\operatorname{trace} Q=\sum_{i=1}^{2 n} \operatorname{trace}\left(d_{p} \Phi_{i}\right)^{2}=\sum_{i=1}^{2 n}\left\|d_{p} \Phi_{i}\right\|^{2} \leq \sum_{i=1}^{2 n} \frac{1}{2}=n
$$


since $\left\|d_{p} \Phi_{i}\right\| \leq 1 / \sqrt{2}$ for all $i=1, \ldots, 2 n$. Hence

$$
\operatorname{det} Q \leq\left(\frac{1}{n} \operatorname{trace} Q\right)^{n} \leq 1 .
$$

Here $\Phi_{i}, i=1, \ldots, n$, are the coordinate functions of $\Phi$ (that is, $\Phi_{i}=\varphi_{e_{i}}$ or $\left.\Phi_{i}=\varphi_{v_{i-n}}\right)$ and all traces and determinants are with respect to the Euclidean structure on $T_{p} M$ defined by the Riemannian metric. The last inequality means that the $n-$ dimensional Jacobian of $\Phi$ at $p$ is no greater than 1 , hence $\Phi$ does not increase $n$-dimensional volumes.

Our next goal is to show that $\Phi(M)$ is contained in a small neighborhood of the subspace $I\left(\mathbb{R}^{n}\right)$ in $\mathbb{R}^{2 n}$ (cf Lemma 3.4). The following lemma is an intermediate step towards this.

Lemma 3.3 For every fixed $r>0$, one has $\operatorname{vol}\left(\Phi^{-1}\left(\mathbb{R}^{2 n} \backslash U_{r}\left(I\left(\mathbb{R}^{n}\right)\right)\right)\right)<\varepsilon(\delta)$.

Proof Denote $W=I\left(\mathbb{R}^{n}\right)$. There is a 1-Lipschitz map $P: \mathbb{R}^{2 n} \rightarrow W$ and a constant $c>0$ such that $\left.P\right|_{I(D)}=\mathrm{id}_{I(D)}$ and

$$
J_{n} P(x) \leq 1-c \quad \text { for all } x \in \mathbb{R}^{2 n} \backslash U_{r}(W),
$$

where $J_{n}$ denotes the $n$-dimensional Jacobian. Indeed, let $Q \subset \mathbb{R}^{2 n}$ be a solid ellipsoid such that $I(D) \subset Q \subset U_{r / 2}(W)$ and let $P_{0}: \mathbb{R}^{2 n} \rightarrow Q$ be the nearest-point projection to $Q$. Then $P_{0}$ is $1-$ Lipschitz and satisfies (3-14) for some $c>0$. A desired map $P$ can be obtained by composing $P_{0}$ with the orthogonal projection to $W$.

Define a map $f: M \rightarrow \mathbb{R}^{n}$ by $f=I^{-1} \circ P \circ \Phi$. Note that $f$ is volume nonincreasing since so are $\Phi, P$ and $I^{-1}$. Let $E=\Phi^{-1}\left(\mathbb{R}^{2 n} \backslash U_{r}(W)\right)$, then (3-14) implies that

$$
\operatorname{vol}(f(E)) \leq(1-c) \operatorname{vol}(E) .
$$

By (3-13) we have $\Phi\left(M \backslash M^{\prime}\right) \subset U_{4 n \delta}(W)$, hence $E \subset M^{\prime}$ provided that $\delta<r / 4 n$. By (3-12), we have $f \approx F$ on $\partial M$. Similarly to the proof of (3-5), this and the fact that $f$ is volume nonincreasing imply that

$$
\operatorname{vol}(f(E))>\operatorname{vol}(E)-\varepsilon(\delta) .
$$

Now the two above inequalities on $\operatorname{vol}(f(E))$ imply that $\operatorname{vol}(E)<\varepsilon(\delta) / c=\varepsilon(\delta)$.

Lemma 3.4 $\Phi(M) \subset U_{\varepsilon(\delta)}\left(I\left(\mathbb{R}^{n}\right)\right)$.

Proof Suppose the contrary. Then there exists $r>0$ such that for every $\delta>0$ there is a manifold $M$ satisfying the assumptions of Theorem 2 and maps $\Phi$ and $I$ constructed as above such that $\operatorname{dist}\left(\Phi(p), I\left(\mathbb{R}^{n}\right)\right) \geq r$ for some $p \in M$. 
Choose such $M, \Phi, I$ and $p$ for a sufficiently small $\delta$. We may assume that $r>8 n \delta$. Consider a metric ball $B=B_{r / 4 n}(p)$. Since $\Phi$ is $2 n$-Lipschitz, we have

$$
\Phi(B) \subset B_{r / 2}(\Phi(p)) \subset \mathbb{R}^{2 n} \backslash U_{r / 2}\left(I\left(\mathbb{R}^{n}\right)\right) .
$$

Therefore $\operatorname{vol}(B)<\varepsilon(\delta)$ by Lemma 3.3, and the 3rd assumption of Theorem 2 implies that $r<\varepsilon(\delta)$, a contradiction.

Now let $P_{i}: \mathbb{R}^{2 n} \rightarrow \mathbb{R}, i=1, \ldots, 2 n$, denote the coordinate projections multiplied by $\sqrt{2}$. Observe that $\varphi_{v}=P_{n+1} \circ \Phi$ and $L_{v}=P_{n+1} \circ I$. Define $P: \mathbb{R}^{2 n} \rightarrow \mathbb{R}^{n}$ by $P=\left(P_{1}, \ldots, P_{n}\right)$, then $P \circ I=\mathrm{id}_{\mathbb{R}^{n}}$ and $P \circ \Phi=\varphi$. By Lemma 3.4, for a given $x \in M$ there is a point $x^{\prime} \in \mathbb{R}^{n}$ such that $\left|\Phi(x)-I\left(x^{\prime}\right)\right|<\varepsilon(\delta)$. Then

$$
\left|\varphi(x)-x^{\prime}\right|=\left|P(\Phi(x))-P\left(I\left(x^{\prime}\right)\right)\right| \leq \sqrt{2}\left|\Phi(x)-I\left(x^{\prime}\right)\right|<\varepsilon(\delta)
$$

where the first inequality follows from the fact that $P$ is $\sqrt{2}$-Lipschitz. Hence

$$
\left|L_{v}(\varphi(x))-L_{v}\left(x^{\prime}\right)\right| \leq\left|\varphi(x)-x^{\prime}\right|<\varepsilon(\delta) .
$$

Furthermore,

$$
\left|\varphi_{v}(x)-L_{v}\left(x^{\prime}\right)\right|=\left|P_{n+1}(\Phi(x))-P_{n+1}\left(I\left(x^{\prime}\right)\right)\right| \leq \sqrt{2}\left|\Phi(x)-I\left(x^{\prime}\right)\right|<\varepsilon(\delta) .
$$

The last two inequalities yield (3-7). This completes the proof of Proposition 3.1.

\section{Estimating distances in $M$}

In this section we finish the proof of Theorem 2 by showing that $\varphi$ almost preserves the distances (up to an additive term $\varepsilon(\delta)$ ).

Lemma 4.1 $|\varphi(x)-\varphi(y)|<d_{M}(x, y)+\varepsilon(\delta)$ for all $x, y \in M$.

Proof Let $v$ be a unit vector in $\mathbb{R}^{n}$ such that $\varphi(x)-\varphi(y)$ is a nonnegative multiple of $v$. Then

$$
|\varphi(x)-\varphi(y)|=L_{v}(\varphi(x))-L_{v}(\varphi(y)) \approx \varphi_{v}(x)-\varphi_{v}(y) \leq d_{M}(x, y) .
$$

Here the first relation follows from the definition of $L_{v}$, the second from (3-7), and the third from the fact that $\varphi_{v}$ is 1 -Lipschitz.

Lemma $4.2 \operatorname{dist}_{M}(x, \partial M) \approx \operatorname{dist}_{\mathbb{R}^{n}}(\varphi(x), \partial D)$ for all $x \in M$. 
Proof Fix $x \in M$. Lemma 4.1 implies that

$$
\left.\operatorname{dist}_{M}(x, \partial M)>\operatorname{dist}_{\mathbb{R}^{n}}(\varphi(x), \varphi(\partial M))-\varepsilon(\delta)>\operatorname{dist}_{\mathbb{R}^{n}}(\varphi(x), \partial D)\right)-\varepsilon(\delta)
$$

since $\varphi(\partial M)$ is contained in a small neighborhood of $\partial D(\operatorname{cf}(3-6))$.

To prove the opposite inequality, let $p \in \partial D$ be a point of $\partial D$ nearest to $\varphi(x)$ and $v$ the inner normal to $\partial D$ at $p$ (or, if $\partial D$ has no tangent hyperplane at $p$, a normal to any supporting hyperplane). If $\varphi(x) \in D$, then $\varphi(x)-p$ is a nonnegative multiple of $v$ and therefore

$$
L_{v}(\varphi(x))=L_{v}(p)+|p-\varphi(x)|=L_{v}(p)+\operatorname{dist}_{\mathbb{R}^{n}}(\varphi(x), \partial D)
$$

by the definition of $L_{v}$. If $\varphi(x) \notin D$, then $\operatorname{dist}_{\mathbb{R}^{n}}(\varphi(x), \partial D) \approx 0$ by (3-6), hence $\varphi(x) \approx p$ and $L_{v}(\varphi(x)) \approx L_{v}(p)$. In both cases we have

$$
\varphi_{v}(x) \approx L_{v}(\varphi(x)) \approx L_{v}(p)+\operatorname{dist}_{\mathbb{R}^{n}}(\varphi(x), \partial D)
$$

where the first relation follows from (3-7). By (3-3) and (3-7),

$$
\varphi_{v}(x)=\varphi_{v}(y)+d_{M}(x, y) \approx L_{v}(\varphi(y))+d_{M}(x, y)
$$

for some $y \in \partial M$. Since $D$ is convex, $p$ is a point of minimum of $\left.L_{v}\right|_{\partial D}$. Since $\varphi(y)$ is close to $\partial D$ (by (3-6)), it follows that $L_{v}(\varphi(y))>L_{v}(p)-\varepsilon(\delta)$. Thus

$$
\varphi_{v}(x)>L_{v}(p)+d_{M}(x, y)-\varepsilon(\delta) \geq L_{v}(p)+\operatorname{dist}_{M}(x, \partial M)-\varepsilon(\delta) .
$$

This and (4-1) imply that $\operatorname{dist}_{M}(x, \partial M)<\operatorname{dist}_{\mathbb{R}^{n}}(\varphi(x), \partial D)+\varepsilon(\delta)$.

Lemma 4.3 For every $r>0$ there is a $\delta_{0}>0$ such that the following holds: if $\delta<\delta_{0}$, $x, y \in M,|\varphi(x)-\varphi(y)| \leq r$ and $\operatorname{dist}_{\mathbb{R}^{n}}(\varphi(x), \partial D) \geq 3 r$, then there is a curve $\gamma$ connecting $x$ and $y$ in $M$ such that $\varphi(\gamma) \subset B_{2 r}(\varphi(x))$.

Proof We may assume that $2 n \delta<r$. Let $B=B_{2 r}(\varphi(x))$. Since $\varphi\left(M \backslash M^{\prime}\right) \subset$ $U_{2 n \delta}(\partial D)(\operatorname{cf}(3-10))$, we have $B \cap \varphi\left(M \backslash M^{\prime}\right)=\varnothing$, hence the set $U:=\varphi^{-1}(B)$ is contained in $M^{\prime}$. Let $U_{x}$ and $U_{y}$ be the connected components of $U$ containing $x$ and $y$, respectively. If $U_{x}=U_{y}$ then any curve $\gamma$ connecting $x$ and $y$ in $U$ satisfies the desired condition.

Suppose that $U_{x} \neq U_{y}$. Since $\varphi$ is $n$-Lipschitz, we have $B_{r / n}(x) \subset U_{x}$. Hence, by the 3rd assumption of Theorem 2, $\operatorname{vol}\left(U_{x}\right) \geq c r^{n}$ where $c=\lambda n^{-n}$. Consider $\varphi_{x}=\left.\varphi\right|_{U_{x}}$ regarded as a map from $U_{x}$ to $B$. This map is proper and hence has a well-defined degree $\bmod 2$. If $\operatorname{deg}_{2}\left(\varphi_{x}\right)=0$, then every regular value of any smooth approximation 
of $\varphi_{x}$ has zero or at least two preimages. Since $\varphi_{x}$ is volume nonincreasing, it follows that

$$
\operatorname{vol}\left(\varphi\left(U_{x}\right)\right) \leq \frac{1}{2} \operatorname{vol}\left(U_{x}\right) \leq \operatorname{vol}\left(U_{x}\right)-c r^{n} / 2,
$$

contrary to (3-5). Thus $\operatorname{deg}_{2}\left(\varphi_{x}\right)=1$.

The same argument applies to $U_{y}$ and a map $\varphi_{y}=\left.\varphi\right|_{U_{y}}: U_{y} \rightarrow B$, thus $\operatorname{deg}_{2}\left(\varphi_{y}\right)=1$ as well. Hence both $\varphi_{x}$ and $\varphi_{y}$ are surjective, hence

$$
\operatorname{vol}(\varphi(U))=\operatorname{vol}\left(\varphi\left(U_{y}\right)\right) \leq \operatorname{vol}\left(U_{y}\right) \leq \operatorname{vol}(U)-\operatorname{vol}\left(U_{x}\right) \leq \operatorname{vol}(U)-c r^{n},
$$

contrary to (3-5).

Lemma 4.4 For every $r>0$ there exist $\rho>0$ and $\delta_{0}>0$ such that the following holds. If $\delta<\delta_{0}$ and $x, y \in M$ are such that $|\varphi(x)-\varphi(y)|<\rho$, then $d_{M}(x, y)<r$.

In other words, $d_{M}(x, y) \rightarrow 0$ as $|\varphi(x)-\varphi(y)| \rightarrow 0$ and $\delta \rightarrow 0$.

Proof Suppose that $r, \rho>0$ and $x, y \in M$ are such that $d_{M}(x, y) \geq r$ and $\mid \varphi(x)-$ $\varphi(y) \mid<\rho$. We are going to obtain a contradiction assuming that $\rho=c r$ for a suitable constant $c>0$ and $\delta \ll r$.

First consider the case when $\operatorname{dist}_{\mathbb{R}^{n}}(\varphi(x), \partial D) \geq 3 \rho$. By Lemma 4.3 there is a curve $\gamma$ connecting $x$ and $y$ such that $\varphi(\gamma) \subset B_{2 \rho}(\varphi(x))$. Let $N$ be a positive integer such that $N \geq n r / \rho>N-1$. Then there are points $x_{1}, \ldots, x_{N}$ on $\gamma$ such that $d_{M}\left(x, x_{k}\right)=(k-1) \rho / n$ for all $k$. The triangle inequality implies that the balls $B_{k}:=B_{\rho / 2 n}\left(x_{k}\right)$ are disjoint. Denote $U=\bigcup B_{k}$. We may assume that $\rho / 2 n>2 \delta$, then by the third assumption of Theorem 2 we have

$$
\operatorname{vol}(U)=\sum \operatorname{vol}\left(B_{k}\right) \geq N \cdot \lambda(\rho / 2 n)^{n}=\mu N \rho^{n}
$$

where $\mu=\lambda(2 n)^{-n}$. Since $\varphi$ is $n$-Lipschitz, we have $\varphi\left(B_{k}\right) \subset B_{\rho / 2}\left(\varphi\left(x_{k}\right)\right)$. Since $\varphi\left(x_{k}\right) \in \varphi(\gamma) \subset B_{2 \rho}(\varphi(x))$ for all $k$, it follows that $\varphi(U) \subset B_{5 \rho / 2}(\varphi(x))$, hence $\operatorname{vol}(\varphi(U)) \leq C \rho^{n}$ where $C$ is the volume of a Euclidean $n$-ball of radius 5/2. Furthermore, $\varphi(U)$ is separated away from $\partial M$ by distance $\rho / 2>2 n \delta$, hence $U \subset M^{\prime}$ $(\operatorname{cf}(3-10))$ and therefore $\operatorname{vol}(\varphi(U))>\operatorname{vol}(U)-\varepsilon(\delta)$ by (3-5). Thus

$$
C \rho^{n} \geq \operatorname{vol}(\varphi(U))>\operatorname{vol}(U)-\varepsilon(\delta) \geq \mu N \rho^{n}-\varepsilon(\delta) \geq \mu r \rho^{n-1}-\varepsilon(\delta)
$$

since $N \geq r / \rho$. Fix $\rho=\mu r / 2 C$ and assume that $\delta$ is so small that the above $\varepsilon(\delta)$ satisfies $\varepsilon(\delta)<\frac{1}{2} \mu r \rho^{n-1}$. Then $C \rho^{n}>\frac{1}{2} \mu r \rho^{n-1}$, hence $r<2 C \rho / \mu=r$, a contradiction. 
It remains to consider the case when $\operatorname{dist}_{\mathbb{R}^{n}}(\varphi(x), \partial D)<3 \rho$. Let $x^{\prime}$ and $y^{\prime}$ be points of $\partial M$ nearest to $x$ and $y$ respectively. Then Lemma 4.2 implies that

$$
d_{M}\left(x, x^{\prime}\right)=\operatorname{dist}_{M}(x, \partial M) \approx \operatorname{dist}_{\mathbb{R}^{n}}(\varphi(x), \partial D)<3 \rho
$$

and

$$
\begin{aligned}
d_{M}\left(y, y^{\prime}\right) & =\operatorname{dist}_{M}(y, \partial M) \\
& \approx \operatorname{dist}_{\mathbb{R}^{n}}(\varphi(y), \partial D) \leq \operatorname{dist}_{\mathbb{R}^{n}}(\varphi(x), \partial D)+|\varphi(x)-\varphi(y)|<4 \rho .
\end{aligned}
$$

Since $\varphi$ is $n$-Lipschitz, it follows that

$$
\left|\varphi(x)-\varphi\left(x^{\prime}\right)\right|<3 n \rho+\varepsilon(\delta) \quad \text { and }\left|\varphi(y)-\varphi\left(y^{\prime}\right)\right|<4 n \rho+\varepsilon(\delta),
$$

hence $\left|\varphi\left(x^{\prime}\right)-\varphi\left(y^{\prime}\right)\right|<|\varphi(x)-\varphi(y)|+7 n \rho+\varepsilon(\delta) \leq(7 n+1) \rho+\varepsilon(\delta)$.

By (3-4),

$$
d_{M}\left(x^{\prime}, y^{\prime}\right) \approx\left|\varphi\left(x^{\prime}\right)-\varphi\left(y^{\prime}\right)\right|<(7 n+1) \rho+\varepsilon(\delta) .
$$

Therefore

$$
r \leq d_{M}(x, y) \leq d_{M}\left(x, x^{\prime}\right)+d_{M}\left(x^{\prime}, y^{\prime}\right)+d_{M}\left(y, y^{\prime}\right)<(7 n+8) \rho+\varepsilon(\delta) .
$$

This is impossible if $\rho \leq \frac{1}{2}(7 n+8)^{-1} r$ and $\delta \ll r$.

Lemma 4.5 $d_{M}(x, y) \approx|\varphi(x)-\varphi(y)|$ for all $x, y \in M$.

Proof By Lemma 4.1, it suffices to show that

$$
d_{M}(x, y)<|\varphi(x)-\varphi(y)|+\varepsilon(\delta) .
$$

Without loss of generality assume that $\operatorname{dist}_{\mathbb{R}^{n}}(\varphi(x), \partial D) \geq \operatorname{dist}_{\mathbb{R}^{n}}(\varphi(y), \partial D)$. Let $v$ be a unit vector in $\mathbb{R}^{n}$ such that $\varphi(x)-\varphi(y)$ is a nonnegative multiple of $v$. By (3-3) there is a point $z \in \partial M$ such that $d_{M}(x, z)=\varphi_{v}(x)-\varphi_{v}(z)$. Let $\gamma$ be a shortest path from $x$ to $z$ in $M$ and $q$ an arbitrary point on $\gamma$, then $d_{M}(x, z)=d_{M}(x, q)+d_{M}(q, z)$. Since $\varphi_{v}$ is $1-$ Lipschitz, we have $\varphi_{v}(x)-\varphi_{v}(q) \leq d_{M}(x, q)$ and $\varphi_{v}(q)-\varphi_{v}(z) \leq d_{M}(q, z)$. If any of these two inequalities is strict, adding them yields that $\varphi_{v}(x)-\varphi_{v}(z)<d_{M}(x, z)$, contrary to the choice of $z$. Thus $\varphi_{v}(x)-\varphi_{v}(q)=d_{M}(x, q)$.

Lemma 4.1 implies that $d_{M}(x, q)>|\varphi(x)-\varphi(q)|-\varepsilon(\delta)$. By (3-7) we have

$$
d_{M}(x, q)=\varphi_{v}(x)-\varphi_{v}(q) \approx L_{v}(\varphi(x))-L_{v}(\varphi(q))=\langle\varphi(x)-\varphi(q), v\rangle,
$$

therefore $\langle\varphi(x)-\varphi(q), v\rangle>|\varphi(x)-\varphi(q)|-\varepsilon(\delta)$. This implies that the vector $\varphi(x)-\varphi(q)$ is $\varepsilon(\delta)$-close to a positive multiple of $v$. Since $q$ is an arbitrary point on $\gamma$, this means that $\varphi(\gamma)$ is contained in an $\varepsilon(\delta)$-neighborhood of the ray $R:=$ $\{\varphi(x)-t v: t \geq 0\}$. 
Since $z \in \partial M, \varphi(z)$ is close to $\partial D$ (cf (3-6)). Since the curve $\varphi(\gamma)$ connects $\varphi(x)$ to $\varphi(z) \in U_{\varepsilon(\delta)}(\partial D), \varphi(\gamma) \subset U_{\varepsilon(\delta)}(R), \varphi(y) \in R \cap \varphi(M) \subset R \cap U_{\varepsilon(\delta)}(D)$ and $D$ is convex, there are two possibilities: either $\varphi(x)$ is close to $\partial D$ or $\varphi(\gamma)$ passes near $\varphi(y)$. In the former case $\varphi(y)$ is close to $\partial D$ as well (by our initial assumption), and the desired assertion follows from Lemma 4.2 and (3-4). In the latter case consider a point $q \in \gamma$ such that $|\varphi(q)-\varphi(y)|<\varepsilon(\delta)$. Since $q \in \gamma$, we have $d_{M}(x, q)<$ $|\varphi(x)-\varphi(q)|+\varepsilon(\delta)$ by (4-2) By Lemma 4.4, the inequality $|\varphi(q)-\varphi(y)|<\varepsilon(\delta)$ implies that $d_{M}(q, y)<\varepsilon(\delta)$, therefore

$$
d_{M}(x, y) \approx d_{M}(x, q)<|\varphi(x)-\varphi(q)|+\varepsilon(\delta) \approx|\varphi(x)-\varphi(y)|
$$

and the lemma follows.

Proof of Theorem 2 By Lemma 4.5, $\varphi$ is an $\varepsilon(\delta)$-approximation of $\varphi(M) \subset \mathbb{R}^{n}$, hence $d_{\mathrm{GH}}(M, \varphi(M))<\varepsilon(\delta)$. By (3-6), the Hausdorff distance in $\mathbb{R}^{n}$ between $\varphi(M)$ and $D$ is small, hence $d_{\mathrm{GH}}(\varphi(M), D)<\varepsilon(\delta)$. Therefore

$$
d_{\mathrm{GH}}(M, D) \leq d_{\mathrm{GH}}(M, \varphi(M))+d_{\mathrm{GH}}(\varphi(M), D)<\varepsilon(\delta)
$$

and the theorem follows.

\section{Proof of Theorem 1}

Let $M$ be a compact Riemannian $n$-manifold with boundary. We use the notation introduced in Section 2.2, namely $S M$ denotes the unit tangent bundle of $M, S_{p}^{+} M$ (where $p \in \partial M$ ) is the hemisphere of inward-pointing vectors from $S_{p} M, \gamma_{v}$ is the maximal forward geodesic with initial velocity vector $v \in S M$ and $\ell(v)$ or $\ell_{M}(v)$ is the length of $\gamma_{v}$. Clearly $\ell$ is a lower semicontinuous function from $S M$ to $[0,+\infty]$.

We say that a unit-speed curve $\gamma:[a, b] \rightarrow M$ is minimizing (or a minimizer, or a shortest path) if it realizes the distance between $\gamma(a)$ and $\gamma(b)$. Since $M$ is compact, every pair of points is connected by a minimizer. Note that a minimizer is not necessarily a geodesic since it may bend along the boundary.

We need some basic facts about minimizers in Riemannian manifolds with boundary (see eg Alexander, Berg and Bishop [1]): every minimizer is $C^{1}$ and (point-wise) convergence of minimizers implies convergence of their tangents.

If two points $x, y \in M$ are such that all shortest paths from $x$ to $y$ have the same velocity vector at $x$, we denote this vector by $\overrightarrow{x y}$ and say that $\overrightarrow{x y}$ is uniquely defined. 
Lemma 5.1 If $b d_{M}$ is differentiable at a point $(x, y) \in \partial M \times \partial M$, then $\overrightarrow{x y}$ is uniquely defined and the projection of $\overrightarrow{x y}$ to $T_{x} \partial M$ equals the Riemannian gradient of the function $-b d_{M}(\cdot, y)$.

Proof This is standard. Denote $f=b d_{M}(\cdot, y)$ and let $\gamma$ be a shortest path from $x$ to $y$. Then the first variation formula implies that for every $v \in T_{x} \partial M$ one has $d_{x} f(v) \leq-\langle v, \dot{\gamma}(0)\rangle$. Applying this to $v$ and $-v$ yields that $d_{x} f(v)=-\langle v, \dot{\gamma}(0)\rangle$ for all $v \in T_{x} \partial M$. Hence the gradient of $-f$ at $x$ is the projection of $\dot{\gamma}(0)$ to $T_{x} \partial M$. Since $\dot{\gamma}(0) \in S_{x}^{+} M$, this vector is uniquely determined by its projection to $T_{x} \partial M$.

Lemma 5.2 If $b d_{M}$ is differentiable away from the diagonal, then every geodesic starting at the boundary is minimizing. In particular, all such geodesics have length bounded above by $\operatorname{diam}(M)$.

Proof Let $\gamma:[0, a] \rightarrow M$ be a geodesic with $\gamma(0)=p \in \partial M$. First consider the case when the initial vector $v:=\dot{\gamma}(0)$ is not tangent to $\partial M$.

Define a map $f: \partial M \backslash\{p\} \rightarrow S_{p}^{+} M$ by $f(x)=\overrightarrow{p x}$. By the previous lemma, this map is well-defined and hence continuous. It is easy to see that

$$
\left|f(x)-u\left(\exp _{p, \partial M}^{-1}(x)\right)\right| \rightarrow 0 \quad \text { as } x \rightarrow p,
$$

where $u: T_{p} M \backslash\{0\} \rightarrow S_{p} M$ is the normalization function defined by $u(w)=w /|w|$, and $\exp _{p, \partial M}$ is the Riemannian exponential map of $\partial M$ at $p$ (restricted to a neighborhood of the origin where it is injective). Denote $\alpha=\angle(v, \partial M)$ and let $B$ be a small geodesic ball in $\partial M$ centered at $p$ such that the left-hand side of (5-1) is less than $\alpha$ for all $x \in B$. Then $\left.f\right|_{\partial B}$ is homotopic to $\left.u \circ \exp _{p, \partial M}^{-1}\right|_{\partial B}$ as a map from $\partial B$ to $S_{p}^{+} M \backslash\{v\}$. Since $\left.u \circ \exp _{p, \partial M}^{-1}\right|_{\partial B}$ is a diffeomorphism from $\partial B$ to the boundary of $S_{p}^{+} M$, it follows that $f$ has degree 1 over $v$. In particular, $f^{-1}(v)$ is nonempty. Therefore there is a point $q \in \partial M$ such that $v=\overrightarrow{p q}$. Then $\gamma$ is an interval of a shortest path from $p$ to $q$ and hence a minimizer.

Now consider the case when $v$ is tangent to the boundary. Choose a sequence $\left\{v_{i}\right\}$ in the interior of $S_{p}^{+} M$ such that $v_{i} \rightarrow v$. As shown above, the geodesics $\gamma_{v_{i}}$ are minimizing. A limit of a subsequence of $\left\{\gamma_{v_{i}}\right\}$ is a minimizer with endpoints at the boundary and with initial velocity $v$. Hence $\gamma$ is an interval of this limit, therefore it is minimizing.

Now assume that $M$ satisfies the assumptions of Theorem 2 for a small $\delta>0$. We continue using the notation $\varepsilon(\delta)$ and $\approx$ defined in Section 3 . 
First observe that the induced Riemannian metric on $\partial M$ at a point $p \in \partial M$ can be recovered from the first derivatives of a function $b d_{M}(p, \cdot)$ near $p$. Indeed, for every tangent vector $v \in T_{p} \partial M$ and a smooth curve $\gamma:[0,1] \rightarrow \partial M$ with $\dot{\gamma}(0)=v$ one has

$$
|v|=\lim _{t \rightarrow 0} d_{\gamma(t)} b d_{M}(p, \cdot)(\dot{\gamma}(t))
$$

where $|\cdot|$ is the norm defined by the Riemannian metric and $d_{\gamma(t)}$ denotes the derivative at $\gamma(t)$. This formula depends continuously on the derivatives of $b d_{M}$, hence

$$
\left\|g_{\left.M\right|_{T \partial D}}-g_{\left.\mathbb{R}^{n}\right|_{T \partial D}}\right\|_{C^{0}} \leq \varepsilon(\delta)
$$

where $g_{M}$ denotes the metric tensor of $M$.

Lemma 5.3 Every nonminimizing geodesic stays within distance $\varepsilon(\delta)$ from $\partial M$.

Proof By Lemma 5.2, a nonminimizing geodesic never hits the boundary and therefore can be extended to infinite length. Let $\gamma$ be a geodesic parametrized by $[0,+\infty)$ and $p=\gamma(0)$; we are to prove that $\operatorname{dist}_{M}(p, \partial M)<\varepsilon(\delta)$. Consider the set $Z$ of all vectors $v \in S_{p} M$ such that the geodesic $\gamma_{v}$ eventually hits $\partial M$. By Lemma 5.2, lengths of these geodesics are bounded above by $\operatorname{diam}(M)$, therefore $Z$ is closed. Obviously $Z \neq \varnothing$, and $Z \neq S_{p} M$ since $\dot{\gamma}(0) \notin Z$. Hence the topological boundary of $Z$ in $S_{p} M$ is nonempty. Let $v \in Z$ be a vector from this boundary. Then $\gamma_{v}$ is tangent to $\partial M$ at its endpoint $q=\gamma_{v}(\ell(v))$. Extend $\gamma_{v}$ backwards until it hits the boundary at a point $s \in \partial M$. (By Lemma 5.2, the backward extension cannot have infinite length since it starts at $q \in \partial M$.)

Since $\overrightarrow{q s}$ is tangent to the boundary, Lemma 5.1 implies that $\left\|d_{q} b d_{M}(s, \cdot)\right\|=1$ where the norm is taken with respect to the metric of $\partial M$. Since $b d_{D}$ is $C^{1-c l o s e}$ to $b d_{M}$ and the metric tensors of $M$ and $D$ are $C^{0}$-close at the boundary, it follows that $\left\|d_{q} b d_{D}(s, \cdot)\right\| \approx 1$ where the norm is taken with respect to the Riemannian metric on $\partial D$ induced from $\mathbb{R}^{n}$. Applying Lemma 5.1 to $D$ yields that the straight line segment $[q s]$ forms almost zero angle with $\partial D$ and hence $|q-s|<\varepsilon(\delta)$. Thus

$$
d_{M}(q, s) \approx d_{D}(q, s) \approx 0 .
$$

Since $p$ lies on a shortest path from $q$ to $s$ in $M$, this implies that

$$
\operatorname{dist}_{M}(p, \partial M) \leq d_{M}(q, p)<d_{M}(q, s)<\varepsilon(\delta)
$$

and the lemma follows.

We are going to show that $M$ satisfies the assumptions of Theorem 2 for $\varepsilon(\delta)$ in place of $\delta$. The first assumption in Theorem 2 is satisfied trivially. 
Denote the value $\varepsilon(\delta)$ from Lemma 5.3 by $\rho$ and let $M^{\prime}=M \backslash U_{\rho}(\partial M)$. Lemma 5.3 implies that all geodesics in $M^{\prime}$ are minimizing. Hence the injectivity radius at every point $x \in M^{\prime}$ is no less than $\operatorname{dist}_{M}\left(x, \partial M^{\prime}\right)$. This fact and Proposition 2.4 imply that

$$
\operatorname{vol}\left(B_{r}(x)\right) \geq c(n) r^{n}
$$

for all $x \in M^{\prime}$ and $r \leq \operatorname{dist}_{M}\left(x, \partial M^{\prime}\right)$. If $r \geq 2 \rho$ and $B_{r}(x) \cap \partial M=\varnothing$, we have $x \in M^{\prime}$ and $\operatorname{dist}\left(x, \partial M^{\prime}\right) \geq r / 2$, hence

$$
\operatorname{vol}\left(B_{r}(x)\right) \geq \operatorname{vol}\left(B_{r / 2}(x)\right) \geq c(n)(r / 2)^{n}=2^{-n} c(n) r^{n} .
$$

Thus $M$ satisfies the third requirement of Theorem 2 for $2 \rho$ in place of $\delta$ and $\lambda=$ $2^{-n} c(n)$.

In order to estimate the volume of $M^{\prime}$ we use Santaló's formula (Proposition 2.2). Let $V_{M} \subset S M$ be the set of all unit tangent vectors $v$ such that the geodesic $\gamma_{-v}$ eventually hits $\partial M$. Applying Proposition 2.2 to the set $A=\bigcup_{p \in \partial M} S_{p}^{+} M$ yields

$$
\mu_{L}\left(V_{M}\right)=\int_{\partial M} d \operatorname{vol}_{\partial M}(p) \int_{S_{p}^{+} M} \ell(v) \cos \angle(v, v(p)) d \operatorname{vol}_{S_{p} M}(v)
$$

where $v(p)$ is the inner normal to $\partial M$ at $p$. Let us compare the inner integral (for a fixed $p \in \partial M$ ) with the similar integral for $D$. Let $I: T_{p} M \rightarrow T_{p} D$ be a linear isometry which preserves the tangent space to the boundary and is $\varepsilon(\delta)$-close to the identity on it. (Such a map exists since the metric tensors of $M$ and $D$ are close to each other on $\partial D$.) Let $v \in S_{p}^{+} M \backslash T_{p} \partial M$ and $q=\gamma_{v}(\ell(v))$. By Lemma 5.1, the $\partial M$-gradient of $-b d_{M}(q, \cdot)$ at $p$ equals the projection of $v$ to $T_{p} \partial M$. Let $v^{\prime}=I(v) \in S_{p}^{+} D \backslash T_{p} \partial D$ and $q^{\prime}$ be the point where the ray $\left\{p+t v^{\prime}: t>0\right\}$ intersects $\partial D$. Note that $v$ and $v^{\prime}$ form the same angle with the inner normals to $\partial M$ and $\partial D$ respectively. By Lemma 5.1 applied to $D$, the $\partial D$-gradient of $-b d_{D}\left(q^{\prime}, \cdot\right)$ at $p$ equals the (Euclidean) projection of $v^{\prime}$ to $T_{p} \partial D$. By the choice of $I$, this projection is close to the above projection of $v$, therefore $d_{p} b d_{M}(q, \cdot) \approx d_{p} b d_{D}\left(q^{\prime}, \cdot\right)$. Since $b d_{M}$ is $C^{1}-$ close to $b d_{D}$, we also have $d_{p} b d_{M}(q, \cdot) \approx d_{p} b d_{D}(q, \cdot)$.

Thus $d_{p} b d_{D}(q, \cdot) \approx d_{p} b d_{D}\left(q^{\prime}, \cdot\right)$. These two derivatives determine the Euclidean directions from $p$ to $q$ and $q^{\prime}$ by means of Lemma 5.1. This implies that $q \approx q^{\prime}$ and therefore

$$
\ell_{M}(v)=d_{M}(p, q) \approx|p-q| \approx\left|p-q^{\prime}\right|=\ell_{D}\left(v^{\prime}\right) .
$$

This and (5-2) imply that $\mu_{L}\left(V_{M}\right) \approx \mu_{L}\left(V_{D}\right)$. Observe that

$$
\mu_{L}\left(V_{D}\right)=\omega_{n-1} \operatorname{vol}(D)
$$


where $\omega_{n-1}$ is the volume of the unit sphere in $\mathbb{R}^{n}$, and

$$
\omega_{n-1} \operatorname{vol}\left(M^{\prime}\right) \leq \mu_{L}\left(V_{M}\right) \leq \omega_{n-1} \operatorname{vol}(M)
$$

since $S M^{\prime} \subset V_{M} \subset S M$. It follows that

$$
\operatorname{vol}\left(M^{\prime}\right) \leq \frac{\mu_{L}\left(V_{M}\right)}{\omega_{n-1}} \approx \frac{\mu_{L}\left(V_{D}\right)}{\omega_{n-1}}=\operatorname{vol}(D),
$$

thus $M^{\prime}$ satisfies the second requirement of Theorem 2 with $\varepsilon(\delta)$ in place of $\delta$.

Thus $M$ satisfies the three requirements of Theorem 2 for $\varepsilon(\delta)$ in place of $\delta$ and some $\lambda$ depending only on $n$. Hence $d_{\mathrm{GH}}(M, D)<\varepsilon(\varepsilon(\delta))=\varepsilon(\delta)$ by Theorem 2 . This completes the proof of Theorem 1.

\section{Concluding remarks and open questions}

\subsection{Regions in $\mathbb{H}^{n}$}

Combining the proof in this paper with technique from $[7 ; 6]$, one can generalize the theorems to the case when $D$ is a region in $\mathbb{H}^{n}$, or, more generally, a region with a Riemannian metric $C^{3}$-close to the Euclidean or the hyperbolic one. To prove these generalizations, replace the map $\varphi$ in Section 3 and the map $f$ in the proof of Lemma 3.3 by area-contracting maps constructed in [6]. (The construction in [6] is in many ways similar to the one in the proof of (3-7); however it uses an auxiliary map to $L^{\infty}\left(S^{n-1}\right)$ rather than $\mathbb{R}^{2 n}$.)

\subsection{Removal of the convexity assumption}

It is interesting whether one can remove the assumption that $D$ is convex. Convexity of $D$ is used in Section 4 and in Lemma 5.3. The former seems easy to work around but the latter presents more of a problem. Estimating the total volume by means of Santaló's formula would not work if a significant portion of the unit tangent bundle is covered by geodesics that never hit the boundary. On the other hand, typical examples where such geodesics are present have nonsmooth boundary distance functions. This raises the following question.

Question 6.1 Let $M$ be a compact Riemannian manifold with nonempty boundary whose boundary distance function is differentiable away from the diagonal. Is it true that $M$ is nontrapping (that is, there are no geodesics of infinite length)?

By Lemma 5.2, an affirmative answer would imply that all geodesics in $M$ are minimizing. Then one could ask whether the same is true for all locally minimizing curves (ie geodesics of the length metric rather than Riemannian geodesics). 


\subsection{Relaxing the ball volume assumption}

Another interesting question is whether the third assumption in Theorem 2 can be replaced by the following: every metric ball of radius $r$ in $M$ (sufficiently separated away from the boundary) is contractible within a ball of radius $\rho(r)$ where $\rho:[0,+\infty) \rightarrow[0,+\infty)$ is a fixed function such that $\rho(r) \rightarrow 0$ as $r \rightarrow 0$. As shown in [11], volumes of $r$-balls (separated away from the boundary) in such $M$ are uniformly bounded below by $v=v_{\rho}(r)>0$. This is similar to the third assumption of Theorem 2 except that $v_{\rho}(r)$ is not of the form $\lambda r^{n}$. (This form of a volume bound is used in Lemma 4.4.)

It is easy to see that a class of Riemannian manifold with a uniform lower bound on volumes of balls depending only on radius, and uniformly bounded diameter and total volume, is precompact in Gromov-Hausdorff topology. Therefore a sequence of manifolds satisfying assumptions (1) and (2) of Theorem 2 and the above uniform local contractibility assumption, must have a partial Gromov-Hausdorff limit. One could try to equip this limit with a structure allowing one to analyze the equality case in the Besicovitch inequality. Such a structure would certainly have applications beyond this particular question.

\section{References}

[1] S B Alexander, ID Berg, R L Bishop, The Riemannian obstacle problem, Illinois J. Math. 31 (1987) 167-184 MR869484

[2] M Berger, Une borne inférieure pour le volume d'une variété riemannienne en fonction du rayon d'injectivité, Ann. Inst. Fourier (Grenoble) 30 (1980) 259-265 MR597027

[3] A S Besicovitch, On two problems of Loewner, J. London Math. Soc. 27 (1952) 141144 MR0047126

[4] G Besson, G Courtois, S Gallot, Entropies et rigidités des espaces localement symétriques de courbure strictement négative, Geom. Funct. Anal. 5 (1995) 731-799 MR1354289

[5] D Burago, Y Burago, S Ivanov, A course in metric geometry, Graduate Studies in Math. 33, Amer. Math. Soc. (2001) MR1835418

[6] D Burago, S Ivanov, Area minimizers and boundary rigidity of almost hyperbolic metrics arXiv:1011.1570

[7] D Burago, S Ivanov, Boundary rigidity and filling volume minimality of metrics close to a flat one, Ann. of Math. (2) 171 (2010) 1183-1211 MR2630062

[8] C B Croke, Some isoperimetric inequalities and eigenvalue estimates, Ann. Sci. École Norm. Sup. (4) 13 (1980) 419-435 MR608287 
[9] C B Croke, B Kleiner, A rigidity theorem for simply connected manifolds without conjugate points, Ergodic Theory Dynam. Systems 18 (1998) 807-812 MR1645381

[10] H Federer, Geometric measure theory, Die Grund. der math. Wissenschaften 153, Springer, New York (1969) MR0257325

[11] R E Greene, P Petersen, V, Little topology, big volume, Duke Math. J. 67 (1992) 273-290 MR1177307

[12] M Gromov, Filling Riemannian manifolds, J. Differential Geom. 18 (1983) 1-147 MR697984

[13] M Gromov, Metric structures for Riemannian and non-Riemannian spaces, Progress in Math. 152, Birkhäuser, Boston (1999) MR1699320 Based on the 1981 French original [MR0682063], With appendices by M Katz, P Pansu and S Semmes, Translated from the French by S M Bates

[14] R Michel, Sur la rigidité imposée par la longueur des géodésiques, Invent. Math. 65 (1981/82) 71-83 MR636880

[15] L Pestov, G Uhlmann, Two dimensional compact simple Riemannian manifolds are boundary distance rigid, Ann. of Math. (2) 161 (2005) 1093-1110 MR2153407

[16] L A Santaló, Integral geometry and geometric probability, Encycl. of Math. and its Appl. 1, Addison-Wesley, Reading, Mass.-London-Amsterdam (1976) MR0433364 With a foreword by $\mathrm{M} \mathrm{Kac}$

[17] P Stefanov, G Uhlmann, Boundary rigidity and stability for generic simple metrics, J. Amer. Math. Soc. 18 (2005) 975-1003 MR2163868

[18] J-N Wang, Stability for the reconstruction of a Riemannian metric by boundary measurements, Inverse Problems 15 (1999) 1177-1192 MR1715358

St.Petersburg Department, Steklov Mathematical Institute RAS

27, Fontanka, 191023 St Petersburg, Russia

svivanov@pdmi.ras.ru

http://eimi.imi.ras.ru/eng/perso/svivanov.php

Proposed: Dmitri Burago

Seconded: Leonid Polterovich, Jean-Pierre Otal
Received: 27 July 2010

Revised: 24 January 2011 